\title{
The AGS High Power Upgrade Plan*
}

\author{
W.T. Weng and T. Roser \\ Brookhaven National Laboratory \\ Upton, NY 11973, USA
}

\begin{abstract}
BNL could provide a Megawatt class neutrino beam from the AGS for very long baseline neutrino experiments. We have studied two possible approaches to upgrade the AGS to $1.0 \mathrm{MW}$ beam power. The first is the linac option, comprising a new superconducting linac injector of $1.2 \mathrm{GeV}$, accelerating $9 \times 10^{13}$ proton per pulse in the AGS to $28 \mathrm{GeV}$ at $2.5 \mathrm{~Hz}$. The second option is to extend the existing $200 \mathrm{MeV}$ linac to $400 \mathrm{MeV}$, ramp the Booster to $2.5 \mathrm{GeV}$ at $6 \mathrm{~Hz}$, add a new $2.5 \mathrm{GeV}$ accumulator ring in the AGS tunnel, and finally ramp the AGS to $28 \mathrm{GeV}$ at 2.5 Hz. Due to the simplicity of the linac approach and minimum interference with the on going research program. the linac option is the preferred one.
\end{abstract}

\section{INTRODUCTION}

We have examined [1] possible upgrades to the AGS complex that would meet the requirements for the proton beam for a $1 \mathrm{MW}$ neutrino super beam facility. Those requirements are summarized in Table 1 and a layout of the upgraded AGS is shown in Figure 1. Since the present number of protons per fill is already close to the required number, the upgrade will focus on increasing the repetition rate and on reducing beam losses to avoid excessive shielding requirements and to maintain the machine components serviceable by hand. It is also important to maintain all the present capabilities of the AGS, in particular its role as injector to RHIC.

We are proposing here to build a superconducting upgrade to the existing $200 \mathrm{MeV}$ Linac to an energy of $1.2 \mathrm{GeV}$ for direct $\mathrm{H}^{-}$injection into the AGS. This will be discussed in the next section. The minimum ramp time to full energy is presently $0.5 \mathrm{~s}$, which will have to be upgraded to reach the required repetition rate of $2.5 \mathrm{~Hz}$. The required upgrade of the AGS power supply will be described in third section. Finally, the increase ramp rate requires a substantial upgrade to the AGS rf system.

TABLE 1. AGS Proton Driver Parameters.

\begin{tabular}{lc}
\hline Total beam power & $1 \mathrm{MW}$ \\
Beam energy & $28 \mathrm{GeV}$ \\
Average beam current & $42 \mu \mathrm{A}$ \\
Cycle time & $400 \mathrm{~ms}$ \\
Number of protons per fill & $9 \times 10^{13}$ \\
Number of bunches per fill & 6 \\
Protons per bunch & $1.6 \times 10^{13}$ \\
Injection turns & 360 \\
Repetition rate & $2.5 \mathrm{~Hz}$ \\
Pulse length & $1.08 \mathrm{~ms}$ \\
Chopping rate & 0.65 \\
Linac average/peak current & $20 / 30 \mathrm{~mA}$ \\
\hline
\end{tabular}

"Work performed under the auspices of the U.S. Department of Energy,
The front end consists of a high-intensity negativeion source, followed by a $750 \mathrm{keV} \mathrm{RFQ}$, and the first 5 tanks of the existing room temperature Drift-Tube Linac (DTL) to accelerate protons to $116 \mathrm{MeV}$. The SCL is made of three sections, each with its own energy range, and different cavity-cryostat arrangement.

The proposed new injector for the AGS is a 1.2 $\mathrm{GeV}$ superconducting linac upgrade with an average output beam power of about $50 \mathrm{~kW}$. The injection energy is still low enough to control beam losses due to stripping of the negative ions that are used for multi-turn injection into the AGS. The duty cycle is about $0.5 \%$. The injection into the AGS is modeled after the SNS [2]. However, the repetition rate and consequently the average beam power is much lower. The larger circumference of the AGS also reduces the number of foil traversals. Beam losses at the injection into AGS are estimated to be about $3 \%$ of controlled losses [1]. The AGS injection parameters are also summarized in Table 1.

\section{SUPERCONDUCTING LINAC}

The superconducting linacs accelerate the proton beam fro, $116 \mathrm{MeV}$ to $1.2 \mathrm{GeV}$. The presented configuration follows a similar design described in detail [3]. All three linacs are built up from a sequence of identical periods.

The major parameters of the three sections of the SCL are given in Table 3. The low energy section operates at $805 \mathrm{MHz}$ and accelerates from 116 to 400 $\mathrm{MeV}$. 
The following two sections, accelerating to 800 $\mathrm{MeV}$ and $1.2 \mathrm{GeV}$ respectively, operate at $1.61 \mathrm{GHz}$. A higher frequency is desirable for obtaining a larger accelerating gradient with a more compact structure and reduced cost.

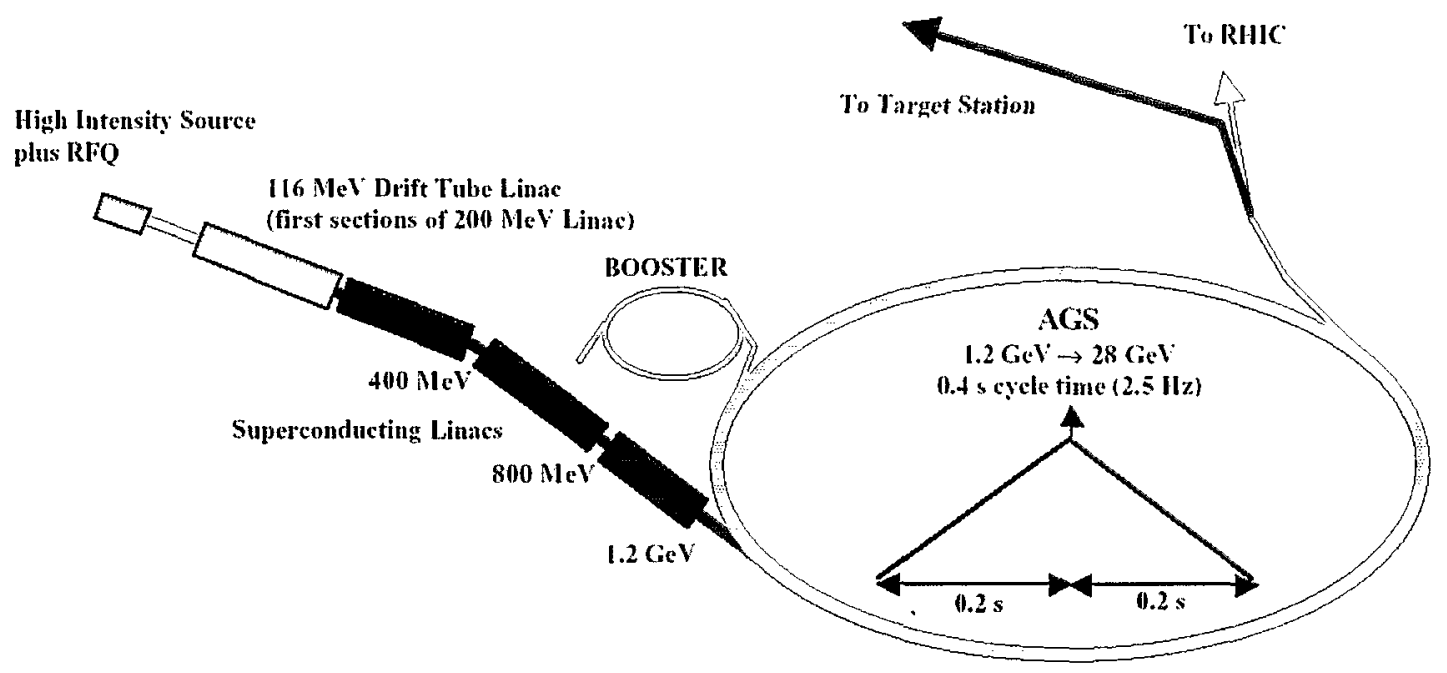

FIGURE 1. AGS Proton Driver Layout.

TABLE 2. Parameters of the Superconducting Linac.

\begin{tabular}{lccc}
\hline & Low & Medium & High \\
\hline Beam Power, $\mathrm{kW}$ & 16 & 32 & 48 \\
Kinetic Energy, MeV & $116-400$ & $400-800$ & $800-1200$ \\
Velocity, c $\beta$ & 0.4560 & 0.7131 & 0.8418 \\
& 0.7131 & 0.8418 & 0.8986 \\
Frequency, MHz & 805 & 1610 & 1610 \\
Protons/ $\mu$ Bunch, $10^{8}$ & 9.32 & 9.32 & 9.32 \\
Temperature, ${ }^{\circ} \mathrm{K}$ & 2.0 & 2.0 & 2.0 \\
Cells/Cavity & 4 & 8 & 8 \\
Cavities/Cryo-Module & 4 & 4 & 4 \\
Cell Length, cm & 9.68 & 6.98 & 8.05 \\
Cell reference velocity, c $\beta_{0}$ & 0.520 & 0.750 & 0.865 \\
Cavity internal diameter & 10 & 5 & 5 \\
Cavity Separation, cm & 32 & 16 & 16 \\
Cold-to-Warm transition & 30 & 30 & 30 \\
Accelerator Gradient, MeV/m & 11.9 & 22.0 & 21.5 \\
Cavities/Klystron & 4 & 4 & 4 \\
No. of Klystrons & 18 & 10 & 9 \\
Klystron Power, $\mathrm{kW}$ & 720 & 1920 & 2160 \\
Energy Gain/Period, MeV & 16.0 & 42.7 & 48.0 \\
Length of a period, m & 4.2 & 4.4 & 4.7 \\
Total length, m & 75.4 & 43.9 & 42.6 \\
\hline
\end{tabular}

of station 1 or 2 through a resistive network. With this grounding configuration, the maximum voltage to ground in the magnets will not exceed 2500 Volt. The magnets are hi-potted to 3000 Volt to ground, prior of each starting of the AGS MMPS after long maintenance periods.

To cycle the AGS ring to $24 \mathrm{GeV}$ at 2.5 pulses per second and with ramp time of 250 $\mathrm{ms}$, the magnet peak current is $4300 \mathrm{Amp}$ and the peak voltage is $25 \mathrm{kV}$. The total average power dissipated in the AGS magnets has been estimated to be $3.7 \mathrm{MW}$. To limit the AGS coil voltage to ground to $2.5 \mathrm{kV}$ the AGS magnets will need to be divided into six identical sections, each powered similarly to present half AGS power system, except that now the magnet loads is $1 / 6$ of the total resistance and inductance.

The peak power required is approximately $110 \mathrm{MW}$ exceeding the $50 \mathrm{MW}$ rating of the existing motor generator. The new motor-generator should also operate with 6 or 12 phases to limit or even eliminate phase-shifting transformers so that every power supply system generates 24 pulses.

\section{AGS RF SYSTEM UPGRADE}

At $2.5 \mathrm{~Hz}$ the peak acceleration rate is three times the present value for the AGS. With 10 accelerating stations each station will need to supply $270 \mathrm{~kW}$ peak power to the beam. The present power amplifier 
TABLE 3. AGS Beam Power With Accumulator

\begin{tabular}{lccc}
\hline & Now & AGS at 1 Hz & AGS at 2.5 Hz \\
\hline Linac Energy (MeV) & 200 & 400 & 400 \\
Booster Intensity (ppp) & $1.5 \times 10^{13}$ & $2.0 \times 10^{13}$ & $2.0 \times 10^{13}$ \\
Booster energy (GeV) & 1.8 & 2.5 & 2.5 \\
Booster Cycles & 4 & 6 & 6 \\
AGS energy (GeV) & 24 & 28 & 28 \\
AGS Intensity & 36 & 120 & 300 \\
AGS Rep Rate (Hz) & 0.6 & 1.0 & 2.5 \\
AGS Current $(\mu \mathrm{A})$ & 5.75 & 19.2 & 48 \\
AGS Intensity $(\mathrm{ppp})$ & $6 \times 10^{13}$ & $12 \times 10^{13}$ & $12 \times 10^{13}$ \\
AGS power (kw) & 138 & 538 & 1344 \\
\hline
\end{tabular}

linac will be improved to inject protons to the booster at $400 \mathrm{MeV}$ (at present it is 200 $\mathrm{MeV}$ ), and the booster energy increased to $2.5 \mathrm{GeV}$ from $1.8 \mathrm{GeV}$. The addition of a fixed field accumulator storage ring in the AGS main tunnel will increase the AGS input beam from the present 4 booster pulses per AGS acceleration to 6 booster pulses per AGS acceleration and, at the same time, increase the AGS frequency from $0.6 \mathrm{~Hz}$ to $1.0 \mathrm{~Hz}$. The AGS power increase would be from 0.14 to $0.53 \mathrm{MW}$. The new accumulator will be in the same tunnel as the AGS. Figure 2 shows the present and proposed AGS injection modes. The AGS intensity upgrades and parameters are shown in Table 3. The fixed field magnets of the accumulator ring will be essentially copies of the permanent magnets produced for a similar purpose at Fermilab [5]. In a second phase of the upgrades the AGS repetition rate will be increased to $2.5 \mathrm{~Hz}$ to reach a total beam power of $1.3 \mathrm{MW}$.

In this way, the injection time of $0.6 \mathrm{sec}$ from the booster to the AGS is reduced to almost zero by prestoring 6 batches of booster beam in the accumulator ring as shown in Figure 2.

The linac option is chosen as the baseline for its simplicity, only involves the linac and the AGS, and the clear upgrade path to $4.0 \mathrm{MW}$ in the future. The accumulator option requires addition/modification of four accelerators, the linac, the booster, the accumulator ring and the AGS. In addition, the installation of the accumulator ring in the AGS tunnel will, inevitably, interference with existing RHIC program.

in the bottom picture the booster will inject 6 pulses into the accumulator which will store the beam until the AGS is at low field and then transfer the beam into the AGS.

\section{REFERENCES}

1. Neutrino Factory Feasibility Study II, http://server.cap.bnl.gov/mumu/studyii/

2. Wei, J., et. al., Low-loss design for the high-intensity accumulator ring of the Spallation Neutron Source, Phys. Rev. ST Accel. Beams 3, 080101 (2000).

3. Ruggiero, A.G., A Superconducting Linac as a New Injector to the BNL AGS, BNL Internal Report, CA/AP/40, February 2001.

4. Neutrino Oscillation Experiments, Letter of Intent to BNL PAC, D. Beavis, M. Diwan, et. al., April 2002.

5. Bill Foster, FNAL, private communication.
FIGURE 2. Time sequence of injecting pulses into the AGS. Top picture shows that at the moment 4 booster pulses are injected into the AGS during the period when the AGS magnets are at low field. In the new proposed configuration 